\title{
Spectral-domain optical coherence tomographic and fundus autofluorescence findings in eyes with primary intraocular lymphoma
}

This article was published in the following Dove Press journal:

Clinical Ophthalmology

31 January 2014

Number of times this article has been viewed

\section{Mariko Egawa \\ Yoshinori Mitamura \\ Yuki Hayashi \\ Takeshi Naito}

Department of Ophthalmology, Institute of Health Biosciences, The University of Tokushima Graduate School, Tokushima, Japan
Correspondence: Mariko Egawa Department of Ophthalmology, Institute of Health Biosciences, The University of Tokushima Graduate School, 3-18-15 Kuramoto, Tokushima 770-8503, Japan Tel +81886337163

Fax $+8 \mid 8$ 863। 4848

Email egawa.m@tokushima-u.ac.jp
Background: The purpose of this study was to evaluate the findings on spectral-domain optical coherence tomography (SD-OCT) and fundus autofluorescence (FAF) in three eyes with primary intraocular lymphoma (PIOL).

Methods: The medical records of three eyes from three patients with biopsy-proven PIOL and retinal infiltrations were reviewed. The SD-OCT and fluorescein angiographic findings were evaluated in the three eyes and FAF images in two eyes.

Results: The PIOL in the three patients was monocular. Vitreous opacities and retinal infiltrations were observed in the three eyes, and iritis was present in two eyes. The cytologic diagnosis was class $\mathrm{V}$ in two eyes and class III in one eye. The interleukin-10/interleukin-6 ratio was $>1.0$ in the vitreous and aqueous humor of the three eyes. The FAF images for two eyes showed abnormal granular hyperautofluorescence and hypoautofluorescence which were the reverse of the pattern in the fluorescein angiographic images. In all three eyes, SD-OCT showed hyper-reflective infiltrations at the level of the retinal pigment epithelium (RPE), a separation of the Bruch membrane from the RPE, damage to the RPE, disruption of the photoreceptor inner segment/outer segment junction, and multiple hyper-reflective signals in the inner retina.

Conclusion: Because of the characteristic FAF and SD-OCT findings in these eyes with PIOL, we suggest that these noninvasive methods may be used for a rapid diagnosis of PIOL and also for understanding the pathology of PIOL.

Keywords: spectral-domain optical coherence tomography, fundus autofluorescence, primary intraocular lymphoma

\section{Introduction}

Primary intraocular lymphomas (PIOLs) are a rare subset of primary central nervous system lymphomas (PCNSLs). They are generally classified as aggressive, diffuse large B-cell lymphomas. It has been reported that $15 \%-25 \%$ of PCNSL cases involve the eye, but PIOLs can be present without central nervous system involvement. ${ }^{1,2}$ Patients with PIOL often present masquerading as uveitis with iritis, vitreous opacities, and retinal infiltrations that delay the diagnosis. In cases that are misdiagnosed as uveitis, the eye may initially respond to corticosteroids which further delays the diagnosis. ${ }^{1,2}$

The prognosis of PIOL remains poor, with a high tendency to progress to PCNSL. Kimura et al reported that the 5-year survival rate of PIOL was $61.1 \%{ }^{3}$ Therefore, it is important to diagnose PIOL promptly. PIOL is diagnosed by cytologic examination of biopsies to identify lymphoma cells, and additional adjunctive diagnosis by measuring interleukin (IL)-10 and IL-6 levels in the intraocular fluids. Eyes with 
PIOL generally have an IL-10/IL-6 ratio greater than 1.0 in intraocular fluid. However, these examinations require invasive biopsy by vitrectomy.

Fundus autofluorescence (FAF) imaging is a noninvasive technique that can acquire a topographic map of the lipofuscin distribution in the retinal pigment epithelium (RPE) cells. Earlier studies have shown that excessive accumulation of lipofuscin in the RPE cells can occur in various retinal diseases, eg, retinitis pigmentosa and age-related macular degeneration..$^{4-7}$ Thus, FAF has been used for diagnosis, determination of pathophysiologic mechanisms, and assessment of the effectiveness of a therapeutic regimen. The FAF and optical coherence tomographic (OCT) findings have recently been reported to be helpful in determining retinal abnormalities in eyes with PIOL. ${ }^{8-12}$

The purpose of this paper is to report pathognomonic FAF and spectral-domain (SD)-OCT findings in eyes with PIOL within a three-patient study. This is important because FAF is a noninvasive technique that could be used for early diagnosis of PIOL.

\section{Patients and methods}

The medical records of three eyes from three patients with biopsy-proven PIOL and retinal infiltrates were reviewed. SD-OCT and fluorescein angiographic images were evaluated in three eyes and FAF images in two eyes. Aqueous humor samples were obtained before vitrectomy, and vitreous samples were obtained during diagnostic vitrectomy. The diagnosis of PIOL was made by detection of malignant lymphocytes, class IV or V, or an IL-10/IL-6 ratio $>1.0$ in the vitreous fluid.

The posterior fundus including areas of infiltration by lymphoma cells into the retina was evaluated by FAF and SDOCT. FAF was performed using a TRC-50DX retinal camera (Topcon, Tokyo, Japan) with an excitation bandpass filter of $535 \mathrm{~nm}$ to $585 \mathrm{~nm}$ and a barrier bandpass filter of $615 \mathrm{~nm}$ to $715 \mathrm{~nm}$. SD-OCT was performed with the Heidelberg Spectralis (Heidelberg Engineering, Heidelberg, Germany). The clinical and imaging characteristics of these three cases are summarized in Table 1.

\section{Case I}

A 57-year-old man visited our clinic with blurred vision in his right eye of 3 months' duration. He had been diagnosed with PCNSL 3 years earlier. Our initial examination showed iritis, mutton fat keratic precipitates, vitreous opacities, retinal infiltrations, and retinal vasculitis. Diagnostic vitrectomy was performed, and cytodiagnosis showed class $\mathrm{V}$

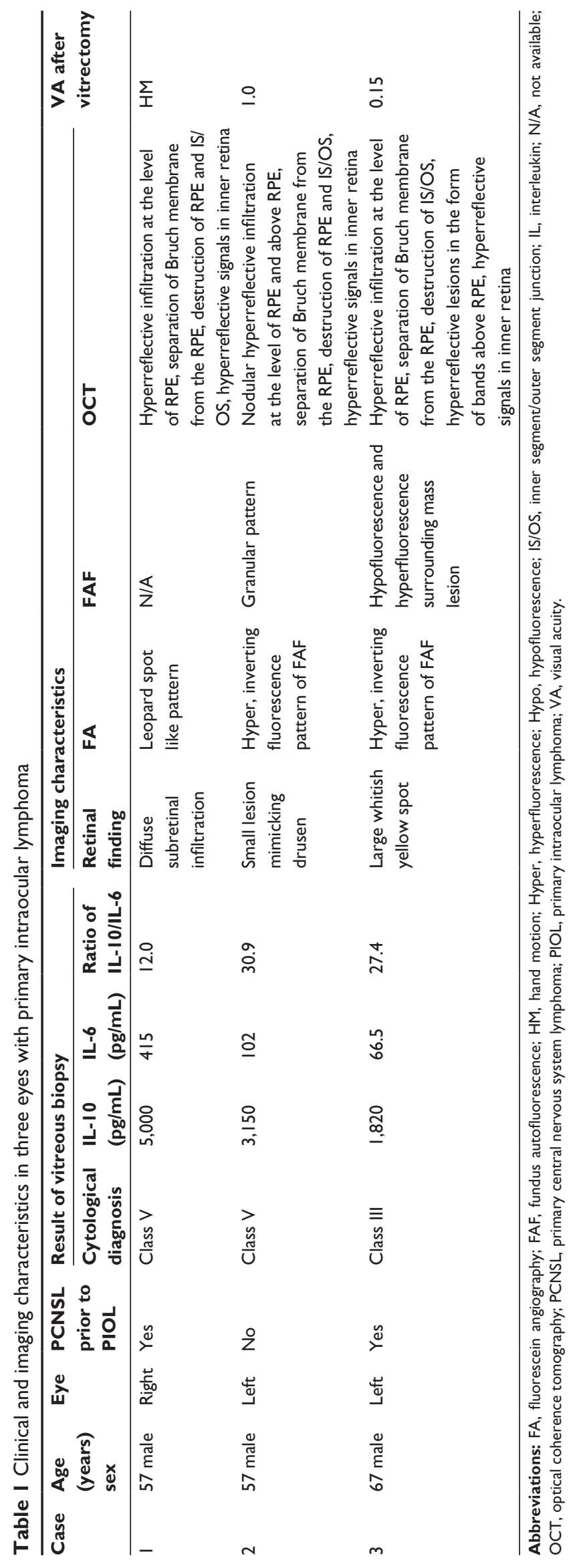


lymphoma cells. The IL-10/IL-6 ratio in the vitreous was 12.0. Thus, a diagnosis of PIOL was made.

His best-corrected visual acuity after vitrectomy was hand motion. Fundus examination revealed diffuse infiltration into the macular area and retinal vasculitis in the temporal and nasal areas (Figure 1A). Late phase fluorescein angiography (FA) showed hypofluorescent spots with a leopard spot-like pattern in the posterior fundus, and staining of the arteries with an avascular area in the nasal area (Figure 1B). SD-OCT examination showed nodular hyper-reflective infiltrations at the level of the RPE, a separation of Bruch's membrane from the RPE, partial destruction of the RPE, disruption of the photoreceptor inner segment/outer segment (IS/OS) junction line, and hyper-reflective signals in the inner retina (Figure 1C). A layered structure of the retina could not be detected on the temporal side. FAF examinations were not performed in this case.

The patient was treated by intravitreal injection of methotrexate. After six injections, the retinal hyper-reflective infiltrations decreased in the SD-OCT images, and the
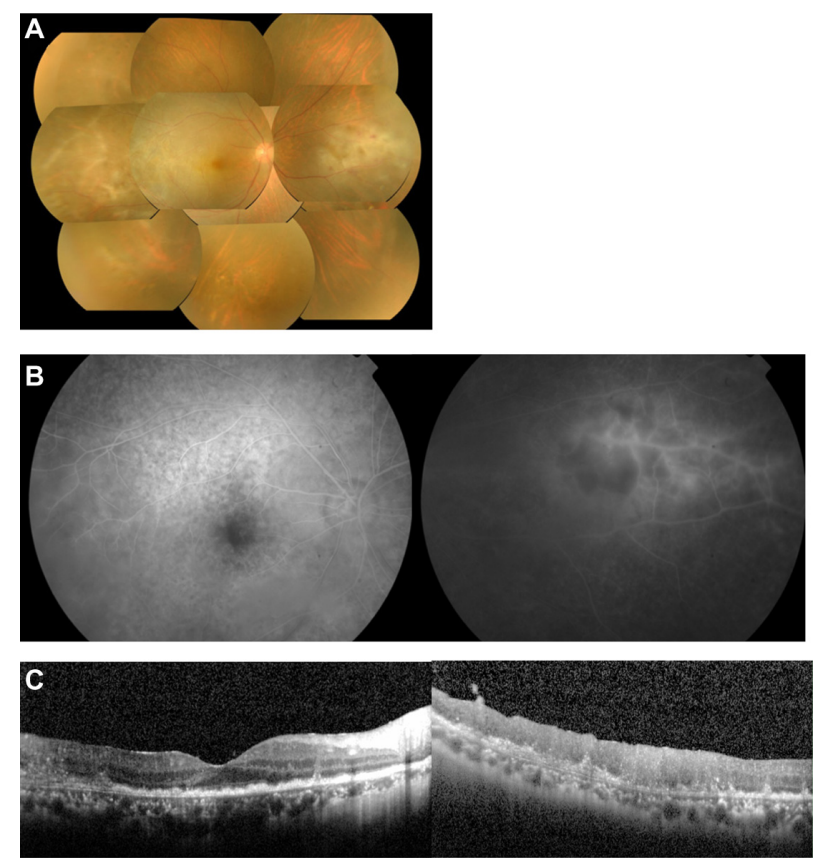

Figure I Case I. Fundus photographs, fluorescein angiograms, and spectral-domain optical coherence tomographic images of the right eye in a 57-year-old man with primary intraocular lymphoma after vitrectomy.

Notes: (A) Color fundus photograph shows diffuse retinal infiltration in the macula and retinal vasculitis in the temporal and nasal areas. (B) Late phase fluorescein angiogram showing hypofluorescent spots with a leopard spot pattern in the posterior fundus and staining of the arteries and an avascular area in the nasal region. (C) Spectral-domain optical coherence tomographic images. (Left) Nodular hyper-reflective infiltration at the level of the retinal pigment epithelium, separation of Bruch's membrane from the retinal pigment epithelium, partial damage of the retinal pigment epithelium, disruption of the photoreceptor inner segment/outer segment junction, and hyper-reflective signals in the inner retina can be seen. (Right) A layered structure of the retina cannot be detected on the temporal side. level of IL-10 in the aqueous humor became undetectable. Recurrence of PCNSL was not found.

\section{Case 2}

A 57-year-old man visited our clinic with blurred vision in his left eye of one month's duration. The initial examination showed diffuse vitreous opacities and retinal infiltrations. Diagnostic vitrectomy was performed, and cytodiagnosis revealed atypical class $\mathrm{V}$ lymphocytes with dyskaryosis and aberrant chromatin (Figure 2B). The IL-10/IL-6 ratio of the vitreous was 30.9. Thus, a diagnosis of PIOL was made.

The best-corrected visual acuity after vitrectomy was 1.0. Fundus examination revealed many small, yellow lesions with distinct boundaries resembling drusen in
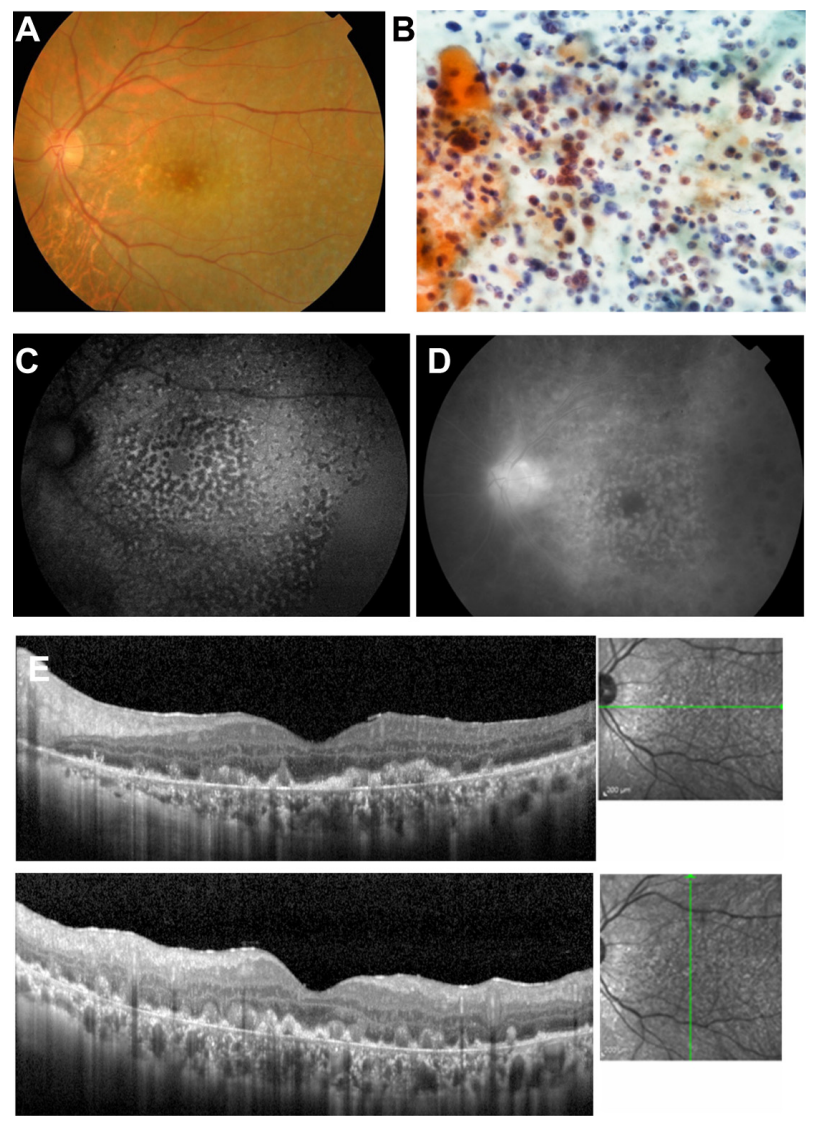

Figure 2 Case 2. Fundus images of the left eye in a 57-year-old man with primary intraocular lymphoma after vitrectomy.

Notes: (A) Color fundus photograph shows many small, yellowish lesions with distinct boundaries resembling drusen in the posterior fundus. (B) Cytological examination of vitrectomy specimen shows atypical lymphocytes with dyskaryosis and aberrant chromatin, stained using May-Grünwald-Giemsa. (C) Granular pattern of hypoautofluorescence and hyperautofluorescence is seen on fundus autofluorescence. (D) Late phase of fluorescein angiography shows hyperfluorescence of the disc and small spots with a reverse fluorescence pattern to the fundus autofluorescence pattern. (E) Spectral-domain optical coherence tomographic images. Nodular hyper-reflective infiltration at the level of retinal pigment epithelium and above the retinal pigment epithelium, a separation of Bruch's membrane from the retinal pigment epithelium, partial destruction of the retinal pigment epithelium, disruption of the photoreceptor inner segment/outer segment junction line, and multiple hyper-reflective signals in the inner retina can be seen. 
the posterior fundus (Figure 2A). A granular pattern of hypoautofluorescence and hyperautofluorescence was seen in the FAF images (Figure 2C). Late phase FA showed hyperfluorescence of the disc and small spots in the posterior fundus that corresponded to the hypofluorescent spots in the FAF images (Figure 2D). SD-OCT showed nodular hyper-reflective infiltrations at and above the level of the RPE, a separation of Bruch's membrane from the RPE, partial destruction of the RPE and IS/OS junction, and multiple hyper-reflective signals in the inner retina (Figure 2E). PCNSL was not detected by magnetic resonance imaging. The patient was treated by intravitreal injection of methotrexate. After four injections, the retinal hyper-reflective infiltrations decreased in the SD-OCT images and the level of IL-10 in the aqueous humor became undetectable.

\section{Case 3}

A 67-year-old man visited our clinic with blurred vision in the left eye of one month's duration. He had been diagnosed with PCNSL one year earlier. Our ophthalmoscopic examination showed diffuse vitreous opacities and retinal infiltrations in the macular area. Diagnostic vitrectomy was performed, and cytodiagnosis revealed class III lymphoma cells. The IL-10/IL-6 ratio in the vitreous was 27.4 , leading to a diagnosis of PIOL.

The decimal best-corrected visual acuity after vitrectomy was 0.15 . Fundus examination revealed many large whitish yellow spots with indistinct boundaries in the macular area (Figure 3A). The FAF images showed hypoautofluorescence and hyperautofluorescence surrounding the spots with a honeycomb shape in the macular area (Figure 3B). Late phase FA showed hyperfluorescence of the disc and a fluorescence pattern of the retina that was the reverse of the FAF pattern (Figure 3C). The SD-OCT images showed hyper-reflective infiltrations at the level of the RPE, separation of Bruch's membrane from the RPE, disruption of the IS/OS junction, hyper-reflective bands above the RPE, and hyper-reflective signals in the inner retina (Figure 3D). Magnetic resonance imaging showed that the PCNSL had not recurred. This patient was treated by intravitreal injection of methotrexate. After six injections, the retinal hyper-reflective infiltrations decreased in the SD-OCT images and the IL-10 level in aqueous humor was undetectable.

\section{Discussion}

The characteristics of the SD-OCT and FAF findings in eyes with PIOL were recently reported, and these imaging techniques were shown to be useful for diagnosing PIOL.
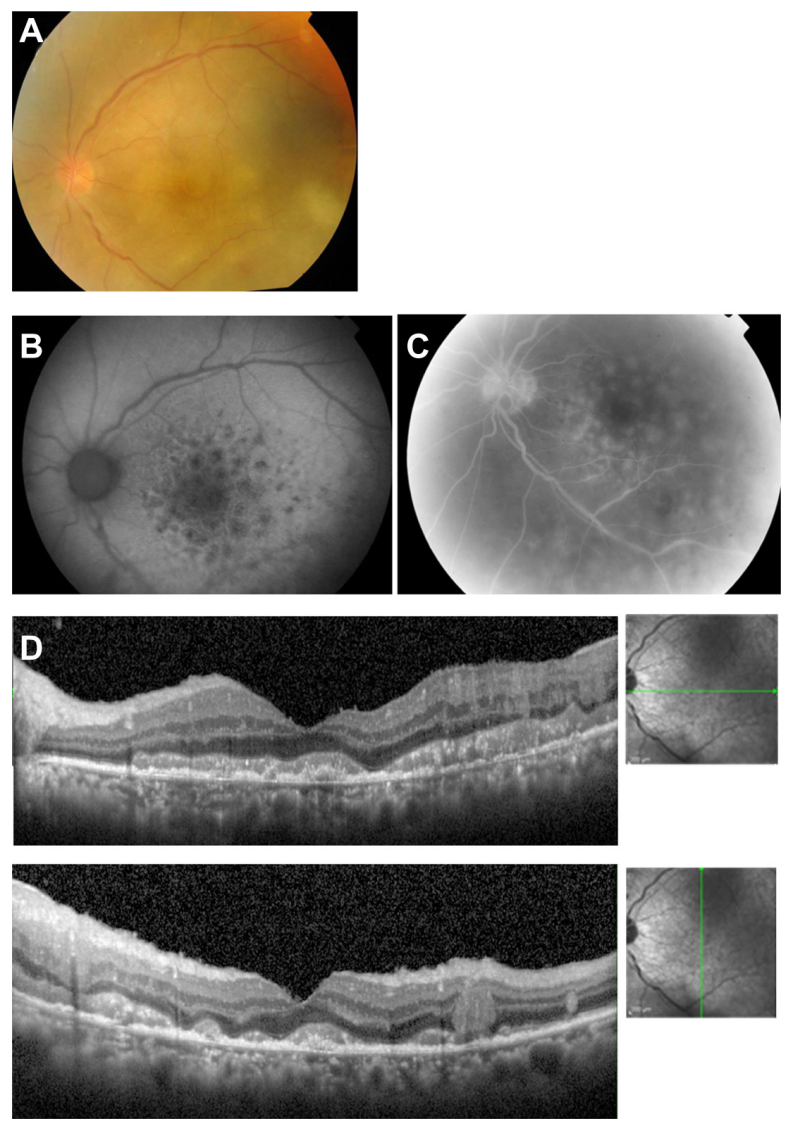

Figure 3 Case 3. Fundus images of the left eye in a 67-year-old man with primary intraocular lymphoma after vitrectomy.

Notes: (A) Fundus photograph showing many large whitish yellow spots with indistinct boundaries in the macular area. (B) Fundus autofluorescence image showing hypoautofluorescence and hyperautofluorescence surrounding a mass lesion with a honeycomb shape. (C) Late phase of fluorescein angiogram shows hyperfluorescence of the disc and fluorescence pattern which is the reverse of the fundus autofluorescence pattern. (D) Spectral-domain optical coherence tomographic image showing hyper-reflective infiltration at the level of the retinal pigment epithelium, separation of Bruch's membrane from the retinal pigment epithelium, disruption of the photoreceptor inner segment/outer segment junction, hyper-reflective lesions in the form of bands above the retinal pigment epithelium, and hyper-reflective signals in the inner retina.

The autofluorescence pattern observed in the FAF images depends on accumulation of lipofuscin, and a normal FAF pattern indicates the presence of intact photoreceptors and normal RPE function. On the other hand, an abnormal hyperautofluorescence level indicates increased metabolic load on the RPE due to degenerated photoreceptor outer segments. A hypoautofluorescent pattern indicates an absence or death of photoreceptor cells following RPE atrophy or the presence of materials that block the FAF signals. Patients with retinitis pigmentosa frequently have an abnormally high parafoveal "autofluorescence ring". This autofluorescence ring is considered to represent the border between functional and dysfunctional retina and is correlated with retinal function and the length of the IS/OS line. ${ }^{5,6}$ 
The active stage of posterior uveitis is often associated with hyperautofluorescent signals in the inflammatory focus, and an inactive stage and chorioretinal atrophy have hypoautofluorescent signals in atrophic lesions. It has been reported that when the hypoautofluorescence includes the fovea, there is a decrease in visual acuity in patients with the white dot syndromes. ${ }^{13}$

It has been reported that one of the characteristic FAF findings in eyes with PIOL is a granular pattern consisting of hyperautofluorescent spots surrounded by a hypoautofluorescent ring. ${ }^{9}$ Casady et al reported that an abnormal autofluorescence pattern in the form of granular hyperautofluorescence and hypoautofluorescence was seen in eleven of 18 eyes (61\%) with PIOL, and that all eyes with a granular FAF pattern had active PIOL at the time of imaging. ${ }^{9}$ In their study, hyperautofluorescent spots in the FAF images were correlated with nodular hyper-reflective spots under the RPE in the OCT images in six eyes. Because FAF depends on the level and distribution of lipofuscin in RPE cells, it was suspected that the areas of lymphomatous infiltration in the sub-RPE space can alter RPE metabolism, leading to hyperautofluorescent FAF. In contrast, a granular pattern, consisting of hypoautofluorescent spots surrounded by hyperautofluorescent rings, was seen in the FAF images in our cases 2 and 3. In these two cases, SD-OCT showed hyper-reflective areas not only under the RPE but also above the RPE. We suggest that lymphomatous infiltration above the RPE might block autofluorescence from the RPE cells. Ishida et al also suggested that the hypoautofluorescence in PIOL patients is caused by RPE atrophy or the blockage of autofluorescence from the RPE by tumor cells. ${ }^{12}$ This blockage of autofluorescence from the RPE by tumor cells might have contributed to the hypoautofluorescent spots seen in our cases 2 and 3 .

It has also been reported that hypofluorescent spots with a leopard spot appearance are the most common FA pattern and these were detected in five of 14 eyes (43\%) with PIOL. ${ }^{9}$ In addition, these hypofluorescent FA spots corresponded to the hyperautofluorescent spots on FAF in all five eyes. In our case 1, the hypofluorescent FA spots also had a leopard spot appearance. It has been suggested that hypofluorescent round spots on FA corresponding to the hyperautofluorescent spots on FAF and nodular hyper-reflective signals under the RPE on OCT may indicate a blockage of choroidal fluorescence on FA by lymphoma cells infiltrating into the sub-RPE space. Velez et al suggested that solid lymphoma masses are made up of tightly packed living tumor cells without fluorescein staining because the membranes of the cells prevent entry of dye into the cytoplasm. ${ }^{14}$ In contrast with these hypofluorescent spots on FA, our cases 2 and 3 had hyperfluorescent spots on FA with a reversed autofluorescence pattern in the FAF pattern. SD-OCT showed hyper-reflective regions not only under the RPE but also above the RPE, and lymphomatous infiltration above the RPE might lead to exudative changes in the retina, which can then lead to hyperfluorescence on FA.

Another distinctive finding in eyes with PIOL was a whitish infiltration into the retina. The areas with infiltration correspond to the hypoautofluorescence of the FAF images and hyperfluorescence of the FA images. ${ }^{9}{ }^{12}$ Casady et al reported that blockage of FAF was seen in two of 18 eyes (11\%) with PIOL. ${ }^{9}$ Ishida et al reported that these white lesions in the OCT images corresponded to diffuse infiltration of tumor cells above the RPE. ${ }^{12}$ It was suggested that the hypoautofluorescence was the result of blocking of autofluorescence by tumor cells above the RPE. Hyperfluorescence on FA may be due to the exudative changes occurring in the retina on invasion by lymphoma cells caused by disruption of the outer blood-retinal barrier. The white lesions around the fovea in our case 3 were hypoautofluorescent in the FAF image and hyperfluorescent in the FA image. This is consistent with earlier reports. ${ }^{9}, 12$ SD-OCT also showed tumor invasion in the form of bands above the RPE.

Intrinsic autofluorescence by malignant B-cells has been studied in vitro. ${ }^{9,15}$ Gastrointestinal lymphoma cells were successfully differentiated from lymphoid hyperplastic cells by autofluorescent imaging. ${ }^{16}$ Casady et al suspected that the intrinsic autofluorescence of lymphocytes or macrophages may also contribute to the abnormal autofluorescence of PIOLs, and that FAF imaging may be useful in differentiating different types of lymphoma. ${ }^{9}$

It was reported that nodular hyper-reflective spots in OCT images were present at the level of the RPE in six of 14 eyes (43\%) with PIOL. ${ }^{9}$ Jang et al reported that hyper-reflective signals were seen as dots, nodules, or bands throughout the retina and RPE in eyes with PIOL. These signals were especially hyper-reflective foci in the inner retina and were characteristic findings of eyes with PIOL. ${ }^{2}$ In that study, the number of hyper-reflective foci increased with an increase in activity of the PIOL. However, hyper-reflective foci have been reported in numerous diseases, including age-related macular degeneration and diabetic macular edema ${ }^{17,18}$ and should be distinguished from findings seen in cases of PIOL. Chan et al reported that lymphoma cells inoculated into the vitreous can migrate and accumulate between the RPE and retina in a murine model of PIOL. ${ }^{19}$ The results of their study 
suggested that lymphoma cells can be found throughout the retina in PIOL cases. Infiltration of lymphoma cells under the RPE causes focal detachments from the RPE because RPE cells are bound by tight junctions and can stretch. We detected hyper-reflective infiltrations at the level of the RPE, and separation of Bruch's membrane from the RPE in the SD-OCT images. These findings are compatible with the histological findings. Several histopathological studies of autopsied eyes from PIOL cases have revealed the presence of malignant cells between the RPE and Bruch's membrane. ${ }^{20,21}$ Further, Liu et al reported that intraretinal and sub-RPE hyper-reflective materials in SD-OCT images were indeed lymphomatous infiltration. ${ }^{8}$ In our cases, destruction of the RPE and IS/OS junction and multiple hyper-reflective signals in the inner retina were also detected on SD-OCT. Disruption of the IS/ OS junction may be related to the visual impairment, although further studies investigating the integrity of the IS/OS junction and visual recovery after treatment will be necessary.

Recent longitudinal studies have documented SD-OCT findings during the progression of PIOL. ${ }^{8,12}$ With progression, hyper-reflective signals appeared in the inner retina, outer retina, and then in the RPE. Although it remains unknown how lymphoma cells migrate into the retina, Chan et al suggested that lymphoma cells migrated from the vitreous to the retina in a murine model of PIOL. ${ }^{19}$ In addition, the hyper-reflective signals have been reported to increase with progression of PIOL. ${ }^{2}$ Thus, SD-OCT may be useful for monitoring the progression or regression of PIOL, which may then influence the management of PIOL cases.

The main limitations of this study are its retrospective nature, lack of control subjects, and small sample size. In addition, FAF was available in only two patients. Further studies examining a larger number of cases with PIOL will be needed to determine more precisely the characteristic FAF and OCT findings of PIOL.

In conclusion, this three-patient study suggests that there may be characteristic FAF and SD-OCT findings in eyes with PIOL. These noninvasive methods can be used for rapid diagnosis of PIOL and understanding of the pathology of this condition.

\section{Acknowledgment}

The authors thank Professor Duco Hamasaki for his critical discussion and final manuscript revision.

\section{Disclosure}

The authors did not receive grant support or research funding for this work and do not have any proprietary interests in materials described in this paper. The authors report no conflicts of interest in this paper.

\section{References}

1. Choi JY, Kafkala C, Foster CS. Primary intraocular lymphoma: a review. Semin Ophthalmol. 2006;21(3):125-133.

2. Jang HS, Sepah YJ, Sophie R, et al. Longitudinal spectral domain optical coherence tomography changes in eyes with intraocular lymphoma. J Ophthalmic Inflamm Infect. 2013;3(1):59.

3. Kimura K, Usui Y, Goto H; Japanese Intraocular Lymphoma Study Group. Clinical features and diagnostic significance of the intraocular fluid of 217 patients with intraocular lymphoma. Jpn J Ophthalmol. 2012;56(4):383-389.

4. Spaide RF. Fundus autofluorescence and age related macular degeneration. Ophthalmology. 2003;110(2):392-399.

5. Aizawa S, Mitamura Y, Hagiwara A, Sugawara T, Yamamoto S. Changes of fundus autofluorescence, photoreceptor inner and outer segment junction line, and visual function in patients with retinitis pigmentosa. Clin Experiment Ophthalmol. 2010;38(6):597-604.

6. Mitamura Y, Mitamura-Aizawa S, Nagasawa T, Katome T, Eguchi H, Naito T. Diagnostic imaging in patients with retinitis pigmentosa. J Med Invest. 2012;59(1-2):1-11.

7. Schmiz-Valckenberg S, Holz FG, Bied AC, Spaide RF. Fundus autofluorescence imaging: review and perspectives. Retina. 2008;28(3): 385-409.

8. Liu TY, Ibrahim M, Bittencourt M, Sepah YJ, Do DV, Nguyen QD. Retinal optical coherence tomography manifestations of intraocular lymphoma. J Ophthalmic Inflamm Infect. 2012;2(4):215-218.

9. Casady M, Faia L, Nazemzadeh M, Nussenblatt R, Chan CC, Sen HN. Fundus autofluorescence patterns in primary intraocular lymphoma. Retina. August 16, 2013. [Epub ahead of print.]

10. Liang F, Barale PO, Hoang Xuan K, Paques M, Sahel JA. Subretinal lymphomatous infiltration in primary intraocular lymphoma revealed by optical coherence tomography. Graefes Arch Clin Exp Ophthalmol. 2011;249(9):1425-1427.

11. Fardeau C, Lee CP, Merle-Béral H, et al. Retinal fluorescein, indocyanine green angiography, and optic coherence tomography in non-Hodgkin primary intraocular lymphoma. Am J Ophthalmol. 2009;147(5):886-894.

12. Ishida T, Ohno-Matsui K, Kaneko Y, et al. Fundus autofluorescence patterns in eyes with primary intraocular lymphoma. Retina. 2010;30(1): 23-32.

13. Yeh S, Forooghian F, Wong WT, et al. Fundus autofluorescence imaging of the white dot syndromes. Arch Ophthalmol. 2010;128(1):46-56.

14. Velez G, Chan CC, Csaky KG. Fluorescein angiographic findings in primary intraocular lymphoma. Retina. 2002;22(1):37-43.

15. Pantanelli SM, Li Z, Fariss R, Mahesh SP, Liu B, Nussenblatt RB. Differentiation of malignant B-lymphoma cells from normal and activated T-cell population by their intrinsic autofluorescence. Cancer Res. 2009;69(11):4911-4977.

16. Ueno N, Fujiya M, Moriichi K, et al. Endosopic autofluorescence imaging is useful for the differential diagnosis of intestinal lymphomas resembling lymphoid hyperplasia. J Clin Gastroenterol. 2011;45: 507-513.

17. Folgar FA, Chow JH, Farsiu S, et al. Spatial correlation between hyperpigmentary changes on color fundus photography and hyperreflective foci on SDOCT in intermediate AMD. Invest Ophthalmol Vis Sci. 2012;53(8):4626-4633.

18. Ota M, Nishijima K, Sakamoto A, et al. Optical coherence tomographic evaluation of foveal hard exudates in patients with diabetic maculopathy accompanying macular detachment. Ophthalmology. 2010;117(10):1996-2002.

19. Chan CC, Fischette M, Shen D, Mahesh SP, Nussenblatt RB, Hochman J. Murine model of primary intraocular lymphoma. Invest Ophthalmol Vis Sci. 2005;46(2):415-459. 
20. Grimm SA, McCannel CA, Omuro AM, et al. Primary CNS lymphoma with intraocular involvement: International PCNSL Collaborative Group Report. Neurology. 2008;71(17):1355-1360.
21. Whitcup SM, Stark-Vancs V, Wittes RE, et al. Association of interleukin 10 in the vitreous and cerebrospinal fluid and primary central nervous system lymphoma. Arch Ophthalmol. 1997;115(9):1157-1160.

\section{Publish your work in this journal}

Clinical Ophthalmology is an international, peer-reviewed journal covering all subspecialties within ophthalmology. Key topics include: Optometry; Visual science; Pharmacology and drug therapy in eye diseases; Basic Sciences; Primary and Secondary eye care; Patient Safety and Quality of Care Improvements. This journal is indexed on

Submit your manuscript here: http://www.dovepress.com/clinical-ophthalmology-journal

\section{Dovepress}

PubMed Central and CAS, and is the official journal of The Society of Clinical Ophthalmology (SCO). The manuscript management system is completely online and includes a very quick and fair peer-review system, which is all easy to use. Visit http://www.dovepress.com/ testimonials.php to read real quotes from published authors. 